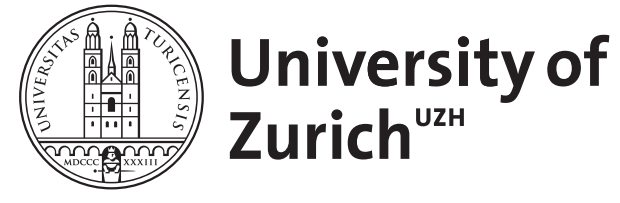
Archive

University of Zurich

University Library

Strickhofstrasse 39

CH-8057 Zurich

www.zora.uzh.ch

Year: 2009

The Zilcha criteria for dynamic inefficiency reconsidered

Barbie, Martin ; Kaul, A

DOI: https://doi.org/10.1007/s00199-008-0367-3

Posted at the Zurich Open Repository and Archive, University of Zurich

ZORA URL: https://doi.org/10.5167/uzh-26709

Journal Article

Published Version

Originally published at:

Barbie, Martin; Kaul, A (2009). The Zilcha criteria for dynamic inefficiency reconsidered. Economic Theory, 40(2):339-348.

DOI: https://doi.org/10.1007/s00199-008-0367-3 


\title{
The Zilcha criteria for dynamic inefficiency reconsidered
}

\author{
Martin Barbie · Ashok Kaul
}

Received: 20 December 2006 / Accepted: 3 April 2008 / Published online: 23 April 2008

(C) Springer-Verlag 2008

\begin{abstract}
We reconsider necessary and sufficient conditions for dynamic inefficiency given in Zilcha (J Econ Theory 52:364-379, 1990, J Econ Theory 55:1-16, 1991) and a critique by Rangazas and Russell (2005). First, we show that the characterization given in Zilcha (1990) for nonstationary economies is correct and correct Zilcha's proof. Second, using this insight, we complement Rangazas and Russell's (Econ Theory 26:701-716, 2005) discussion of the counterexamples to Zilcha (J Econ Theory 55:1-16,1991). Third, we discuss consequences of our results for applied tests of (in-)efficiency based on the Zilcha criteria.
\end{abstract}

Keywords Dynamic inefficiency $\cdot$ Zilcha criterion $\cdot$ Pareto optimality

JEL Classification $\quad \mathrm{D} 51 \cdot \mathrm{D} 90 \cdot \mathrm{E} 13 \cdot \mathrm{E} 22$

We would like to thank Itzhak Zilcha, and in particular Peter Rangazas and Steve Russell for detailed and very helpful comments.

M. Barbie

Department of Economics, University of Karlsruhe, 76128 Karlsruhe, Germany

e-mail: barbie@wior.uni-karlsruhe.de

A. Kaul (ه)

Gutenberg University Mainz,

Department of Economics - FB 03, 55099 Mainz, Germany

e-mail: ashok.kaul@uni-mainz.de

A. Kaul

IEW, University of Zurich, Zurich, Switzerland 


\section{Introduction}

We reconsider characterizations of dynamic (in-) efficiency in competitive stochastic general equilibrium models as well as their observational implications. The analysis focuses on important contributions that extend the Cass (1972) criterion to a stochastic setup for nonstationary [Zilcha (1990)] and stationary economies (Zilcha 1991).

In a recent paper, Rangazas and Russell (2005) criticize Zilcha's characterizations for dynamic inefficiency in overlapping generations models. They come to the conclusion that the general characterization of dynamic inefficiency in stochastic nonstationary economies, the main result in Zilcha (1990), is (1) stated and (2) proved incorrectly and offer a corrected version of this characterization. Contrary to the first claim, we show in this note that the dynamic inefficiency characterization given in Zilcha (1990) is stated correctly and is indeed equivalent to the corrected statement given in Rangazas and Russell (2005).

However, as pointed out by Rangazas and Russell (2005), the proof offered by Zilcha (1990) is incorrect. The Cass-type argument given by Zilcha proves only a weaker result, namely that inefficiency holds if and only if a uniform boundedness condition holds. ${ }^{1}$ This is shown in Rangazas and Russell (2005). Our result shows that this uniform boundedness condition holds if and only if Zilcha's original characterization for nonstationary economies is valid. In Sect. 2, we correct the proof of Zilcha's result.

Rangazas and Russell (2005) present illustrative counterexamples to the Zilcha (1991) criterion for inefficiency in stationary economies. They construct an economy that satisfies his (stationary) inefficiency criterion but for which no improving reallocation of capital exists. They show how the uniform boundedness condition is violated. However, given that Zilcha's (1990) statement of the result for nonstationary economies turns out to be correct, the question arises: What invalidates the test in Zilcha (1991) that is based on the original result from Zilcha (1990)? We investigate this question in Sect. 3 by elaborating on a counterexample given in Rangazas and Russell (2005).

In Sect. 4, we then briefly discuss the consequences of our results for applied tests of (in-)efficiency based on the Zilcha criteria. Our conclusion is that Zilcha's stationary criterion is valid only as a sufficient condition for efficiency, as is already pointed out by Rangazas and Russell (2005), whereas the criterion cannot be used for a test of inefficiency.

\section{Zilcha's characterization for nonstationary economies}

The analysis focuses on contributions to the dynamic efficiency literature within the framework of nonstationary (Zilcha 1990) and stationary stochastic OLG economies (Zilcha 1991). We borrow our notation from Rangazas and Russell (2005). We also refer to their paper for a description of the model.

\footnotetext{
1 See also e.g. Chattopadhyay and Gottardi (1999), whose extension of the Cass type proof to a stochastic environment in the context of a pure exchange economy yields a uniform boundedness condition.
} 
Denote by $S$ the time-invariant shock space (that affects the production function) with generic element $\omega_{t} . S$ is assumed to be a compact Polish space. ${ }^{2}$ Let ${ }^{0} \Omega^{*}$ denote the set consisting of infinite subsequences of the form $\left(\omega_{0}, \omega_{1}, \ldots, \omega_{t}, \ldots\right)$. For each $t \geq 0$, we define ${ }^{0} \mathcal{F}_{t}^{*}$ as the Borel $\sigma$-algebra on ${ }^{0} \Omega^{*}$ generated by cylinder sets of the form $B=\times_{k=0}^{\infty} B_{k}$ with $B_{k} \subseteq S, B_{k}$ is an open set for $0 \leq k \leq t$ and $B_{k}=S$ for $t>k$ (see footnote 4 on p.705 in Rangazas and Russell (2005)). We thus have a measurable space $\left({ }^{0} \Omega^{*},{ }^{0} \mathcal{F}^{*}\right)$ and consider a probability measure $P$ on this space. Denote the set of feasible allocations given the initial capital stock $k_{0}$ by "FPCA from $k_{0}$ " (feasible production consumption allocation).

Since our general shock space allows e.g. the case where $S=[a, b]$ for some positive constants $a$ and $b$, it seems reasonable to require in the case of inefficiency only an improvement (i.e., an increase in aggregate consumption) almost surely at each point in time instead of requiring the improvement in each history $\left(\omega_{0}, \omega_{1}, \ldots, \omega_{t}\right) .{ }^{3}$ In the following, the a.s. qualification is always with respect to the probability measure $P$. With this modification, the condition in Lemma 1 in Zilcha (1990) reads as: The economy is inefficient if and only if for some $t \geq 0$ there exists a set $A$, where $A$ $\epsilon^{0} \mathcal{F}_{t}^{*}$ has strictly positive $P$-measure, and there exists another FPCA with $\widehat{k}$ such that

$$
\begin{gathered}
k_{s}(\omega)=\widehat{k}_{s}(\omega) \text { for } \omega \notin A \text { and all } s \text { a.s. } \\
k_{t+1}(\omega)>\widehat{k}_{t+1}(\omega) \text { for } \omega \in A \\
k_{s+1}(\omega)-\widehat{k}_{s+1}(\omega) \geq f\left(k_{s}(\omega), \omega_{s}\right)-f\left(\widehat{k}_{s}(\omega), \omega_{s}\right) \text { for } \omega \in A, s \geq t+1 \text { a.s. }
\end{gathered}
$$

We now state Zilcha's (1990) characterization of (dynamic) inefficiency in two equivalent forms:

Theorem 1 AFPCA $\left\{k_{s}(\omega)\right\}_{s=1}^{\infty}$ from $k_{0}^{*}$ is inefficient if and only if one of the following equivalent conditions holds.

(a) (Zilcha (1990)) For some $t \geq 0$ there exists a set $\widetilde{A}=A \backslash \bigcup_{s=t+1}^{\infty} N_{s}$, where $A \in{ }^{0} \mathcal{F}_{t}^{*}$ has strictly positive $P$-measure, $N_{s} \in{ }^{0} \mathcal{F}_{s}^{*}$ with $P\left(N_{s}\right)=0$ for each $s=t+1, \ldots$, such that

$$
\sum_{s=t}^{\infty} \frac{1}{\psi_{s}(\omega)}<\infty
$$

for each $\omega \in \widetilde{A}$ where $\psi_{s}(\omega)$ is defined $\psi_{s-1}(\omega)=f^{\prime}\left(k_{s}(\omega), \omega_{s}\right) \psi_{s}(\omega)$, $s=0,1,2, \ldots$, with $\psi_{-1}(\omega) \equiv 1$.

\footnotetext{
2 Rangazas and Russell (2005) use a finite shock space. We can nevertheless use the notation from Rangazas and Russell (2005) and make adaptations where our more general shock space requires this.

3 We thank Steven Russell for pointing out this reasonable extension to us. Zilcha (1990) did not consider the exception of sets of measure zero in his Lemma 1.
} 
(b) For some $t \geq 0$ there exists a set $\widetilde{A}=A \backslash \bigcup_{s=t+1}^{\infty} N_{s}$, where $A \in^{0} \mathcal{F}_{t}^{*}$ has strictly positive $P$-measure, $N_{s} \in{ }^{0} \mathcal{F}_{s}^{*}$ with $P\left(N_{s}\right)=0$ for each $s=t+1, \ldots$, and $a$ constant $B$ such that

$$
\sum_{s=t}^{\infty} \frac{1}{\psi_{s}(\omega)}<B
$$

for each $\omega \in \widetilde{A}$ where $\psi_{s}(\omega)$ is defined $\psi_{s-1}(\omega)=f^{\prime}\left(k_{S}(\omega), \omega_{s}\right) \psi_{s}(\omega)$, $s=0,1,2, \ldots$, with $\psi_{-1}(\omega) \equiv 1$.

First, note that the modification in part (a) and (b) concerning the sets $N_{S}$ come from the modification in Lemma 1 of Zilcha (1990) to require the condition (2) (condition (17 b) on p. 373 in Zilcha (1990)) to hold only a.s., which gives for each $t$ a set $N_{t} \in^{0} \mathcal{F}_{t}^{*}$ with $P\left(N_{t}\right)=0$. In the proof of Theorem 2 in Zilcha (1990), we get the estimate (23) in Zilcha (1990) on $A$ except for $\omega \in \bigcup_{s=t+1}^{\infty} N_{s}$. Similarly, the construction of an improving reallocation of capital done on p. 377 in Zilcha (1990) is carried out on $A$ except for $\omega \in \bigcup_{s=t+1}^{\infty} N_{s}$. Without the a.s. qualification in (1)- the case considered in Zilcha (1990) — the theorem remains the same with the exception that for all zero measure sets we have $N_{s}=\emptyset$.

Second, if we follow the lines of the proof of Theorem 2 in Zilcha (1990), in part (b) there should be (instead of a constant $B$ ) a positive ${ }^{0} \mathcal{F}_{t}^{*}$-measurable function $C(\omega)$ (with $K$ as a positive constant) which has the form $C(\omega)=K /\left[\psi_{t}(\omega)\left(k_{t+1}(\omega)\right.\right.$ $\left.\left.-\widehat{k}_{t+1}(\omega)\right)\right]$. The ${ }^{0} \mathcal{F}_{t}^{*}$-measurability of $C$ ensures that $C(\omega)=C(\widetilde{\omega})$ if $\left(\omega_{0}, \omega_{1}, \ldots, \omega_{t}\right)=\left(\widetilde{\omega}_{0}, \widetilde{\omega}_{1}, \ldots, \widetilde{\omega}_{t}\right)$. By our restriction to interior allocations, $\psi_{t}(\omega)$ is always bounded below, but $k_{t+1}(\omega)-\widehat{k}_{t+1}(\omega)$ could become arbitrarily small for some history $\omega^{t}$, so that there does not necessarily exist a uniform upper bound. However, given that $A \in^{0} \quad \mathcal{F}_{t}^{*}$ has strictly positive measure and that $P(A)=$ $\lim _{n \rightarrow \infty} P\left(\left\{k_{t+1}(\omega)-\widehat{k}_{t+1}(\omega)>\frac{1}{n}\right\}\right)$, we must have $P\left(\left\{k_{t+1}(\omega)-\widehat{k}_{t+1}(\omega)>\frac{1}{n}\right\}\right)>$ 0 for some $n$, and by defining $A^{\prime}=\left\{k_{t+1}(\omega)-\widehat{k}_{t+1}(\omega)>\frac{1}{n}\right\}$, we can assume the uniform bound $B$.

Proof Obviously, (4) implies (3), but the converse is not obvious and requires a proof. Suppose that the condition in part (a) holds and assume that (b) does not hold. Since ${ }^{0} \Omega_{t}^{*}:=S_{0} \times \cdots \times S_{t}$ with $S_{i}=S$ for all $i$ is a metrizable space (as the product of metrizable spaces), by Theorem 12.5 in Aliprantis and Border (2006) the probability measure $P$ restricted to $\left({ }^{0} \Omega_{t}^{*},{ }^{0} \mathcal{F}_{t}^{*}\right)$ is inner regular, ${ }^{4}$ which implies that there exists a closed set $K_{0} \in{ }^{0} \mathcal{F}_{t}^{*}$ such that $P\left(K_{0}\right)>0$ and $K_{0} \subset A$. For each $\omega \in K_{0}$, define $T_{1}(\omega)=\min \left\{T \geq t\right.$ such that $\left.\sum_{s=t}^{T} \frac{1}{\psi_{s}(\omega)}>1\right\}$. Note that $\left\{T_{1} \leq n\right\} \in^{0} \mathcal{F}_{n}^{*}$, since $\left\{T_{1} \leq n\right\}=\cup_{s=t}^{n}\left\{\sum_{i=t}^{s} \frac{1}{\psi_{i}(\omega)}>1\right\} \cap K_{0}$ and $\left\{\sum_{i=t}^{s} \frac{1}{\psi_{i}(\omega)}>1\right\} \cap K_{0} \in^{0} \mathcal{F}_{s}^{*}$. Thus also $\left\{T_{1}=n\right\} \in^{0} \mathcal{F}_{n}^{*}$. If $P\left(\cup_{n=t}^{\infty}\left\{T_{1}=n\right\}\right)=0$, the set $\widehat{A}:=K_{0} \backslash\left(\bigcup_{s=t+1}^{\infty} N_{s} \cup\right.$

\footnotetext{
${ }^{4}$ Strictly speaking, we should endow ${ }^{0} \Omega_{t}^{*}$ with its Borel $\sigma$-algebra ${ }^{0} \mathcal{B}_{t}$. We can; however, identify each set $A \in{ }^{0} \mathcal{B}_{t}$ with the set $A \times S_{t+1} \times S_{t+2} \times \cdots$ from ${ }^{0} \mathcal{F}_{t}^{*}$ and that is what we have done when we consider $\left({ }^{0} \Omega_{t}^{*},{ }^{0} \mathcal{F}_{t}^{*}\right)$.
} 
$\bigcup_{s=t}^{\infty}\left\{T_{1}=s\right\}$ ) would satisfy condition (b) of the theorem (for $B=1$ ). Thus, we must have $P\left(\cup_{n=t}^{\infty}\left\{T_{1}=n\right\}\right)>0$ and thus there must exist some $t_{1} \geq t$ with $P\left(\left\{T_{1}=t_{1}\right\}\right)>0$. Now define $A_{1}:=\left\{T_{1}=t_{1}\right\} \backslash \cup_{j=t+1}^{t_{1}} N_{j} \in \mathcal{F}^{0} \mathcal{F}_{t_{1}}^{*}$. Since ${ }^{0} \Omega_{t_{1}}^{*}$ is a metrizable space (as the product of metrizable spaces), by Theorem 12.5 in Aliprantis and Border (2006) the probability measure $P$ restricted to $\left({ }^{0} \Omega_{t_{1}}^{*},{ }^{0} \mathcal{F}_{t_{1}}^{*}\right)$ is inner regular, which implies there exists a closed set $K_{1} \in \mathcal{F}_{t_{1}}^{*}$ such that $P\left(K_{1}\right)>0$ and $K_{1} \subset A_{1}$. Define $T_{2}(\omega)=\min \left\{T>t_{1}\right.$ such that $\left.\sum_{s=t_{1}+1}^{T} \frac{1}{\psi_{s}(\omega)}>1\right\}$ for $\omega \in K_{1}$. By the same arguments as before, there exists some $t_{2}>t_{1}$ with $P\left(\left\{T_{2}=t_{2}\right\}\right)>0 .{ }^{5}$ Continuing inductively we obtain closed sets $K_{0} \supseteq K_{1} \supseteq K_{2} \supseteq \cdots$ with $P\left(K_{i}\right)>0$. Since the $K_{i}$ are subsets of the compact space ${ }^{0} \Omega^{*}$ (it is compact as the product of compact spaces) and are thus compact themselves. By the finite intersection property, they have a nonempty intersection (see Theorem 2.31 in Aliprantis and Border 2006). Let $\omega^{*} \in \bigcap_{i=0}^{\infty} K_{i}$. By construction, $\sum_{s=t}^{t_{i}} \frac{1}{\psi_{s}\left(\omega^{*}\right)}>i$ for every $i$, and so $\sum_{s=t}^{\infty} \frac{1}{\psi_{s}\left(\omega^{*}\right)}=\infty$. Clearly, $\omega^{*} \in A$. Further, $\omega^{*} \notin \bigcup_{s=t+1}^{\infty} N_{s}$. This holds because for each $s$ there exists some $t_{i}>s$, which by the construction of $K_{i}$ implies $K_{i} \cap N_{s}=\emptyset$. Thus $\omega^{*} \in \widetilde{A}$, and we obtain a contradiction to assumption (a) of the theorem.

Remark 1 The integers $t$ and the sets $\widetilde{A}$ in conditions (a) and (b) can be different.

Remark 2 In the special case of a finite $S$, each set from ${ }^{0} \mathcal{F}_{t}^{*}$ has only a finite number of elements. Further, with a probability measure $P$ such that each time $t$ history $\left(\omega_{0}, \omega_{1}, \ldots, \omega_{t}\right)$ has strictly positive probability, the following holds: $N_{s} \in \in^{0} \mathcal{F}_{s}^{*}$ with $P\left(N_{s}\right)=0$ implies $N_{s}=\emptyset$. Note that the case considered in Rangazas and Russell (2005) in which shocks each period are i.i.d. with a finite support falls into this category.

We thus obtain as a corollary:

Corollary 1 Let $S$ be finite and let $P$ be such that $P\left(\left\{\omega_{0}, \omega_{1}, \ldots, \omega_{t}\right\}\right)>0$ for each $\left(\omega_{0}, \omega_{1}, \ldots, \omega_{t}\right)$. A FPCA $\left\{k_{s}(\omega)\right\}_{s=1}^{\infty}$ from $k_{0}^{*}$ is inefficient if and only if one of the following equivalent conditions holds.

(a) For some $t \geq 0$ there exists a nonempty set $A \in \in^{0} \mathcal{F}_{t}^{*}$ such that

$$
\sum_{s=t}^{\infty} \frac{1}{\psi_{s}(\omega)}<\infty
$$

for each $\omega \in A$ where $\psi_{s}(\omega)$ is defined $\psi_{s-1}(\omega)=f^{\prime}\left(k_{s}(\omega), \omega_{s}\right) \psi_{s}(\omega)$, $s=0,1,2, \ldots$, with $\psi_{-1}(\omega) \equiv 1$.

(b) (Rangazas and Russell (2005)) For some $t \geq 0$ there exists a nonempty set $A \in \in^{0} \mathcal{F}_{t}^{*}$ and a constant $B>0$ such that

$$
\sum_{s=t}^{\infty} \frac{1}{\psi_{s}(\omega)}<B
$$

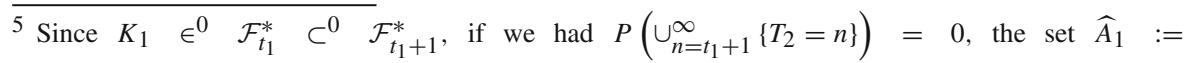
$K_{1} \backslash\left(\bigcup_{s=t_{1}+2} N_{s} \cup \bigcup_{s=t_{1}+1}\left\{T_{2}=s\right\}\right)$ would satisfy condition (b) of the theorem and we had $\sum_{s=t_{1}+1}^{\infty} \frac{1}{\psi_{s}(\omega)} \leq 1$ for all $\omega \in \widehat{A}_{1}$. 
for each $\omega \in A$ where $\psi_{s}(\omega)$ is defined $\psi_{s-1}(\omega)=f^{\prime}\left(k_{s}(\omega), \omega_{s}\right) \psi_{s}(\omega)$, $s=0,1,2, \ldots$, with $\psi_{-1}(\omega) \equiv 1$.

Our result shows that the statement of the characterization of dynamic inefficiency in Theorem 2 of Zilcha (1990) is correct and that the "correction" in Rangazas and Russell (2005) is merely a restatement of the same condition. ${ }^{6}$ However, Rangazas and Russell (2005) are right in pointing out that the proof offered by Zilcha (1990) is incorrect: what is proved in Zilcha (1990) only allows us to conclude that inefficiency is equivalent to the seemingly different condition (b). Additional work is needed to prove the validity of the statement of Theorem 2 in Zilcha (1990). Our proof fills this gap.

\section{Detecting inefficiency in stationary economies}

In a sequel paper, Zilcha (1991) presented a necessary and sufficient condition for dynamic inefficiency of stationary economies based on his characterization in Zilcha (1990), Theorem 2. Rangazas and Russell (2005) present a counterexample to Zilcha (1991) showing that his condition for stationary economies is only necessary, but not sufficient for dynamic inefficiency. They show that the economy does not satisfy condition (6) and conclude correctly that the allocation is efficient. However, they claim incorrectly that the economy satisfies the (seemingly) weaker condition (5). ${ }^{7}$ So given that the statement of Theorem 2 in Zilcha (1990) is correct, what makes the Zilcha (1991) inefficiency characterization for stationary economies fail? It turns out that the restatement of his Theorem 2 from Zilcha (1990) in Zilcha (1991) on p. 7 is incorrect. The restated condition is not necessary and sufficient for dynamic inefficiency any more. We will now investigate these points in detail. ${ }^{8}$

For the ease of notation we dispense with (population) growth, which was introduced in Zilcha (1991). The Zilcha dynamic inefficiency test for stationary economies for a finite $S$ then reads [see Theorem 1 in Zilcha (1991) and p. 706 in Rangazas and Russell (2005)]:

\footnotetext{
6 In fact, Rangazas and Russell (2005) make their statement of condition (a) and (b) (and also of Lemma 1 of Zilcha (1990)) only for sets $A$ of positive measure from the $\sigma$-algebra ${ }^{0} \mathcal{F}_{0}^{*}$, not for sets of positive measure from a $\sigma$-algebra ${ }^{0} \mathcal{F}_{t}^{*}$ for some $t \geq 0$. If one allows for arbitrary nonstationary allocations as in Zilcha (1990), this will in general not be sufficient. Consider e.g. an economy with two possible shock realizations each period. Suppose that we always have $f^{\prime}\left(k(\omega), \omega_{t}\right) \leq m<1$ for $t \geq 2$ if $\omega_{1}=\underline{\omega}$. For the other date 0 shock $\bar{\omega}$ suppose we always have $f^{\prime}\left(k(\omega), \omega_{t}\right)>1$ for $t \geq 2$ if $\omega_{1}=\bar{\omega}$. Then there exists no positive measure set from ${ }^{0} \mathcal{F}_{0}^{*}$ such that (3) holds, but there exists the positive measure set $A=S \times\{\underline{\omega}\}$ from ${ }^{0} \mathcal{F}_{1}^{*}$ where (3) holds. With Lemma 1, p. 370 in Zilcha (1990), one can increase consumption with positive probability without lowering it anywhere, so that the economy is dynamically inefficient. See also the original statement in Theorem 2 of Zilcha (1990), which corresponds exactly to our condition (a).

7 They write on p. 711 of their paper that in the stationary economy they construct, the Zilcha inefficiency criterion for stationary economies and hence (5) is satisfied, while (6) does not hold.

8 See Zilcha (1991) and Rangazas and Russell (2005) for a description of the notion of stationarity employed.
} 
Theorem 2 (Zilcha 1991, Theorem 1) A stationary competitive allocation is dynamically inefficient if and only if

$$
E\{\ln r(\omega)\}<0
$$

holds, where $E\{\ln r(\omega)\}=\int_{\Omega} \ln f^{\prime}\left(k\left(T^{-1} \omega\right), \omega_{0}\right) \mu^{\infty}(d \omega), T$ is the shift operator and $r(\omega)=f^{\prime}\left(k\left(T^{-1} \omega\right), \omega_{0}\right)$.

We now elaborate on an example constructed by Rangazas and Russell (2005) that has the following features: the (finite number of) shocks each period are i.i.d. and the realizations that occur with positive probability are real numbers $\beta=\omega^{1}>\omega^{2}>$ $\cdots>\omega^{s}=\alpha$ with $s \geq 2$ and $\alpha>0$. There is an interval $\left[k_{\alpha}, k_{\beta}\right]$ with $k_{\alpha}, k_{\beta}>0$ and a continuous function $g(k, \omega)$ that is both increasing in $k$ and $\omega$ such that the dynamics of capital accumulation is described by $g$, i.e., $k_{t+1}=g\left(k_{t}, \omega_{t}\right)$. Further $k_{\alpha}=g\left(k_{\alpha}, \alpha\right)$ and $k_{\beta}=g\left(k_{\beta}, \beta\right)$, i.e. if the capital stock starts in $\left[k_{\alpha}, k_{\beta}\right]$, the capital stock will stay there forever. As noted in Rangazas and Russell (2005), the series $\sum_{t=0}^{\infty} \frac{1}{\psi_{t}(\omega)}$ can be rewritten as $\sum_{t=0}^{\infty} \prod_{\tau=0}^{t} f^{\prime}\left(k_{\tau}(\omega), \omega_{\tau}\right)$. The production function $f$ is chosen such that $f^{\prime}(k, \widetilde{\omega})$ is decreasing in $k$ and increasing in $\widetilde{\omega}$. The example is constructed in such a way that $E\left\{\ln f^{\prime}\left(k_{\alpha}, \widetilde{\omega}\right)\right\}<0$, where the expectation is taken with respect to the i.i.d. random variable $\widetilde{\omega}$ distributed on $\omega^{1}>\omega^{2}>\cdots>\omega^{s}$. Given the monotonicity properties of $f^{\prime}$ and that all capital stocks are contained in $\left[k_{\alpha}, k_{\beta}\right]$, this implies that (7) is satisfied, seemingly indicating inefficiency.

A feature of the example is that $f^{\prime}\left(k_{\beta}, \beta\right)>1$, which implies that neither (5) nor (6) holds. To see this, consider for any finite sequence of shocks $\left(\omega_{0}, \omega_{1}, \ldots, \omega_{t}\right)$ the continuation path given by $\omega^{*}=\left(\omega_{0}, \omega_{1}, \ldots, \omega_{t}, \beta, \beta, \beta, \ldots\right)$. Note that

$$
\begin{aligned}
& \sum_{s=0}^{\infty} \prod_{\tau=0}^{s} f^{\prime}\left(k_{\tau}\left(\omega^{*}\right), \omega_{\tau}^{*}\right) \\
& \quad=\sum_{s=0}^{t} \prod_{\tau=0}^{s} f^{\prime}\left(k_{\tau}(\omega), \omega_{\tau}\right)+\prod_{\tau=0}^{t} f^{\prime}\left(k_{\tau}(\omega), \omega_{\tau}\right) \sum_{s=t+1}^{\infty} \prod_{\tau=t+1}^{s} f^{\prime}\left(k_{\tau}\left(\omega^{*}\right), \beta\right) \\
& \quad \geq \sum_{s=0}^{t} \prod_{\tau=0}^{s} f^{\prime}\left(k_{\tau}(\omega), \omega_{\tau}\right)+\prod_{\tau=0}^{t} f^{\prime}\left(k_{\tau}(\omega), \omega_{\tau}\right) \sum_{s=t+1}^{\infty} \prod_{\tau=t+1}^{s} f^{\prime}\left(k_{\beta}, \beta\right) \rightarrow \infty .
\end{aligned}
$$

This implies that each positive measure set $A \in^{0} \mathcal{F}_{t}^{*}$ for some $t \geq 0$ contains a path $\omega$ such that $\sum_{t=1}^{\infty} \frac{1}{\psi_{t}(\omega)}=\infty$ and thus Zilcha's (1990) original inefficiency criterion (5) fails for this economy.

Rangazas and Russell (2005) show that if $f^{\prime}\left(k_{\beta}, \beta\right)>1$ then there is always a positive-measure subset $A \in^{0} \mathcal{F}_{t}^{*}$ on which $\sum_{t=0}^{\infty} \frac{1}{\psi_{t}(\omega)}$ exceeds any particular bound. The argument presented in the proof of Zilcha's (1990) Theorem 2 implies that an allocation is inefficient if and only if there is no subset of this type: that is, if and only if $\sum_{t=0}^{\infty} \frac{1}{\psi_{t}(\omega)}$ is bounded almost surely. So they reached the (incorrect) conclusion that the inefficiency condition from the statement of the theorem is wrong, and that it needed to be replaced by the condition that $\sum_{t=0}^{\infty} \frac{1}{\psi_{t}(\omega)}$ is almost surely 
bounded. As we show, however, this revision of the statement of the theorem turns out to be unnecessary. We show that $\sum_{t=0}^{\infty} \frac{1}{\psi_{t}(\omega)}$ is uniformly finite (i.e. finite for every $\omega \in A$ ), as Zilcha's statement of Theorem 2 requires, if and only if it is uniformly bounded, and that uniform boundedness and almost-sure boundedness are equivalent, in this context.

How does condition (7) from Zilcha (1991) deviate from Zilcha's (1990) original inefficiency condition? To see what Zilcha's stationary economies criterion accomplishes, consider

$$
\prod_{\tau=0}^{t} f^{\prime}\left(k_{\tau}(\omega), \omega_{\tau}\right)=\exp \sum_{\tau=0}^{t} \ln \left[f^{\prime}\left(k_{\tau}(\omega), \omega_{\tau}\right)\right] \leq \exp \sum_{\tau=0}^{t} \ln \left[f^{\prime}\left(k_{\alpha}, \omega_{\tau}\right)\right] .
$$

The random variables $\ln \left[f^{\prime}\left(k_{\alpha}, \omega_{\tau}\right)\right], \tau=0, \ldots, t$ are i.i.d. (and integrable) and thus the strong law of large numbers implies

$$
\frac{1}{t} \sum_{\tau=0}^{t} \ln \left[f^{\prime}\left(k_{\alpha}, \omega_{\tau}\right)\right] \stackrel{t \rightarrow \infty}{\longrightarrow} E\left\{\ln f^{\prime}\left(k_{\alpha}, \widetilde{\omega}\right)\right\}<0 \quad \text { a.s. }
$$

where almost surely (a.s.) refers to the probability measure $\mu^{\infty}$ on the set of paths ${ }^{0} \Omega^{*}$ (and on the $\sigma$-algebra ${ }^{0} \mathcal{F}^{*}$ ) generated by the i.i.d. random variables on the shock space $S$. Note that under the probability measure $\mu^{\infty}$, each single path $\omega \in^{0} \Omega^{*}$ has probability 0 , i.e. $\mu^{\infty}(\{\omega\})=0$. (8) implies that

$$
\prod_{\tau=0}^{t} f^{\prime}\left(k_{\tau}(\omega), \omega_{\tau}\right) \leq \exp \left[\frac{t}{2} E\left\{\ln f^{\prime}\left(k_{\alpha}, \widetilde{\omega}\right)\right\}\right]
$$

a.s. for $t$ sufficiently large and thus $\sum_{t=0}^{\infty}\left(\exp \left[\frac{1}{2} E\left\{\ln f^{\prime}\left(k_{\alpha}, \widetilde{\omega}\right)\right\}\right]\right)^{t}<\infty$ implies $\sum_{t=0}^{\infty} \prod_{\tau=0}^{t} f^{\prime}\left(k_{\tau}(\omega), \omega_{\tau}\right)=\sum_{t=0}^{\infty} \frac{1}{\psi_{t}(\omega)}<\infty$ a.s..

The critical feature of the result is that it only guarantees convergence almost surely and not convergence for every $\omega \in^{0} \Omega^{*}$. The mistake in Zilcha's (1990) proof of Theorem 2 causes the argument to appear to imply that the requirement that $\sum_{t=0}^{\infty} \frac{1}{\psi_{t}(\omega)}$ should be uniformly finite is stronger than necessary, and that it would be enough for $\sum_{t=0}^{\infty} \frac{1}{\psi_{t}(\omega)}$ to be almost surely finite. This is where Zilcha seems to have gone wrong in his 1991 paper (Zilcha 1991). In that paper, he restates Theorem 2 so that the requirement is for $\sum_{t=0}^{\infty} \frac{1}{\psi_{t}(\omega)}$ to be finite almost surely. Later in the paper, he presents criterion (7) and shows that it is equivalent to almost-sure finiteness of $\sum_{t=0}^{\infty} \frac{1}{\psi_{t}(\omega)}$. So he concludes, incorrectly, that (7) is necessary and sufficient for inefficiency. As Rangazas and Russell (2005) counterexample illustrates, almost-sure finiteness of $\sum_{t=0}^{\infty} \frac{1}{\psi_{t}(\omega)}$ is not sufficient for almost-sure boundedness of that sum. So they were able to construct an example in which the inefficiency criterion (7) is satisfied but the allocation is efficient. However, our argument shows that uniform finiteness of $\sum_{t=0}^{\infty} \frac{1}{\psi_{t}(\omega)}$ is necessary and sufficient for uniform boundedness thereof. In the example, $\sum_{t=0}^{\infty} \frac{1}{\psi_{t}(\omega)}$ is 
not uniformly finite. So it is not a counterexample to Theorem 2 of Zilcha (1990), as incorrectly asserted in Rangazas and Russell (2005).

To see how the condition $\sum_{t=0}^{\infty} \frac{1}{\psi_{t}(\omega)}=\infty$ on a set of measure zero interact with the impossibility of "Zilcha reallocation" (as outlined in Rangazas and Russell (2005)), consider paths of the form $\left(\omega_{0}, \omega_{1}, \ldots, \omega_{t}, \beta, \beta, \beta, \ldots\right)$. They form a set $B_{t}^{\infty}$, the set of paths for which after some finite $t$ only the high shock $\beta$ occurs. This set has measure zero. For $T$ define $B_{t}^{T}$ as the set of paths whose first $T$ entries coincide with the first $T$ entries of some member of the set $B_{t}^{\infty}$. For any finite $T$, this set has positive mesure. Consider any $\Lambda>0$. Given that $f^{\prime}(k, \beta) \geq f^{\prime}\left(k_{\beta}, \beta\right)>1$ for all $k \in\left[k_{\alpha}, k_{\beta}\right]$ it follows that given such a $\Lambda$ we can always find a $\widehat{T}$ such that $\sum_{t=0}^{\infty} \frac{1}{\psi_{t}(\omega)}>\Lambda$ for all $\omega \in B_{t}^{\widehat{T}}$. Thus, the paths on which the capital stock vanishes has strictly positive probability and the allocation is dynamically efficient. This is the argument used by Rangazas and Russell (2005), p. 713 to show efficiency. At the same time, for the measure zero set $B_{t}^{\infty}$ the inefficiency condition of Theorem 2 in Zilcha (1990) does not hold. Our Theorem 1 implies that under Zilcha's assumptions, sets like $B_{t}^{\widehat{T}}$ cannot be ruled out without ruling out sets like $B_{t}^{\infty}$.

\section{Consequences for applying (In-)efficiency tests}

How useful are Zilcha's (in-)efficiency characterizations for applied tests of (in-) efficiency? Our analysis showed on the one hand that for nonstationary economies the dynamic inefficiency characterization given in Zilcha (1990) is correct, and in fact, equivalent to one given in Rangazas and Russell (2005). This seems to be good news, because testing whether a series as in (3) converges for every $\omega$ is easier to test than testing whether there is additionally a uniform bound $B$ over all $\omega$ as in (4). This should make it easier to derive econometric tests from the characterization.

On the other hand, for stationary economies, there seems to be no way to construct tests of inefficiency based on some sort of law of large numbers as done in Zilcha (1991). More generally, all statistical/econometric testing procedures usually do not take into account sets of measure zero, i.e. make statements at most "almost surely". 9 In the light of the importance of sets of measure zero in evaluating efficiency of a given allocation, it seems very difficult to come up with such a test.

It should, however, be noted that these problems arise only if the test indicates inefficiency, i.e. with the sufficiency part of the inefficiency criterion. If we have $E\{\ln r(\omega)\}>0$, we can conclude that $\sum_{t=0}^{\infty} \frac{1}{\psi_{t}(\omega)}=\infty$ a.s. with a similar argument as shown above [see the proof of Theorem 1 in Zilcha (1991)]. This is clearly sufficient to conclude dynamic efficiency from (5). In Barbie et al. (2004), we provide some evidence from U.S. economic data that this may be the empirically relevant case.

\footnotetext{
9 The sets of measure zero that are excluded in a testing procedure as described in the previous section are different from those sets of measure zero excluded in Theorem 1. First, Zilcha (1991) has only a finite $S$, so that $N_{s}=\emptyset$ for the measure zero sets from Theorem 1 (compare with Remark 2). Second and more general, if such a test is done for a general shock space, the paths excluded by the test are from ${ }^{0} \mathcal{F}^{*} \backslash \cup_{s=0}^{\infty 0} \mathcal{F}_{s}^{*}$ and are thus different from the sets $N_{s}$ in Theorem 1.
} 
Another aspect of Zilcha's definition of dynamic efficiency is that it rules out gains from risk sharing from different agents and therefore may be of limited empirical relevance. An efficiency concept that takes such considerations into account is e.g. used in the influential paper of Abel et al. (1989). ${ }^{10}$ In Barbie et al. (2004), we construct an example in which $\sum_{t=0}^{\infty} \frac{1}{\psi_{t}(\omega)}=\infty$ a.s., but there are nevertheless efficiency gains by intergenerational risk sharing in the sense of Abel et al. (1989). This fact may help encourage future research on the difficult but important question of dynamic inefficiency.

\section{References}

Abel, A.B., Mankiw, N.G., Summers, L.H., Zeckhauser, R.J.: Assessing dynamic efficiency: theory and evidence. Rev Econ Stud 56, 1-19 (1989)

Aliprantis, C.D., Border, K.C.: Infinite dimensional analysis, 3 edn. Heidelberg: Springer (2006)

Barbie, M., Hagedorn M., Kaul, A.: Assessing aggregate tests of efficiency for dynamic economies. Topics Macroecon 4(1), Article 16 (2004)

Cass, D.: On capital overaccumulation in the aggregative, neoclassical model of economic growth: A complete characterization. J Econ Theory 4, 200-223 (1972)

Chattopadhyay, S., Gottardi, P.: Stochastic OLG models, market structure, and optimality. J Econ Theory 89, 21-67 (1999)

Rangazas, P., Russell, S.: The Zilcha criterion for dynamic inefficiency. Econ Theory 26, 701-716 (2005)

Zilcha, I.: Dynamic efficiency in overlapping generations models with stochastic production. J Econ Theory 52, 364-379 (1990)

Zilcha, I.: Characterizing efficiency in stochastic overlapping generations models. J Econ Theory 55, 1-16 (1991)

10 They also call their their efficiency concept "dynamic efficiency", but it has a different meaning than in Zilcha $(1990,1991)$, namely they use it in the sense of interim Pareto optimality. It distinguishes agents according to the state nature in which they are born. Another efficiency concept is ex ante Pareto optimality, which treats agents born in different states of nature, but at the same time as one person. 